\title{
An Analytic Trajectory Planner for Aircraft with Severe Damage or Failures
}

\author{
Hee Jun Choi* and Ella M. Atkins ${ }^{\dagger}$ \\ University of Michigan, Ann Arbor, MI 48105
}

\begin{abstract}
This paper describes an analytic real-time emergency landing trajectory planner applicable in situations where an aircraft experiences severe damage or failure(s) that contract the feasible lateral plane flight envelope. Solutions are constructed as sequences of spirals that follow a reference arc, extending a traditional Dubins path solver to handle cases in which an aircraft cannot fly straight. A comprehensive Turning Dubins Vehicle (TDV) solver is presented to handle the spectrum of relative distances and headings between aircraft initial state and the landing runway approach end. This solver is shown to generate minimum-distance landing paths. Example solutions are presented with future work aimed at assessing the impact of transitions and disturbances as well as application to specific damage/failure scenarios.
\end{abstract}

\section{Nomenclature}

$\sigma \quad$ Curve for the Turning Dubins Vehicle (TDV)

$\mathcal{O}$ Circular curve for the TDV

a Circular arc curve for the TDV

$b \quad$ Product of circular arc curves

$\Sigma \quad$ Set of possible curves for the TDV

$\Sigma_{c} \quad$ Set of circular curves for the TDV

$\mathcal{A} \quad$ Set of circular arc curves for the TDV

$\mathcal{A}_{r} \quad$ Set of possible reference arcs connecting two centers of the initial and final circular curves

$\mathcal{B} \quad$ Set of possible sequences of two different turning radii for the TDV

$\vec{V} \quad$ Velocity vector

$\vec{K} \quad$ Curvature vector

$r \quad$ Radius of circular curve

$\delta \quad$ Central angle of the reference arc

$\rho \quad$ Central angle of the circular flight path arc

$\tau \quad$ Opposite central angle of the circular flight path arc subtending $2 \pi-\rho$

$l \quad$ Length of flight path arc curves

$l_{J} \quad$ Length of the reference arc traversed over $(n-1)$ arc pair sequences

$J \quad$ Length of the reference arc traversed over two-arc sequence

$n \quad$ Number of arc sequences in $\mathcal{B}$

$n_{m} \quad$ Minimum number of arc sequences in $\mathcal{B}$

$\lambda \quad$ Distance of the points of a straight line from a known point

Subscript

$r \quad$ Reference arc

$1 \quad$ First circular curve of two different radius circular curves

$2 \quad$ Second circular curve of two different radius circular curves

$m \quad$ Minimum radius turning circle

$M \quad$ Maximum radius turning circle

*Research Assistant, Aerospace Engineering, University of Michigan, Ann Arbor, MI 48105

${ }^{\dagger}$ Associate Professor, Aerospace Engineering, University of Michigan, Ann Arbor, MI 48105, Associate Fellow 


\section{Introduction}

Modern aircraft operate safely over a variety of conditions. Flight management systems capably monitor onboard systems and are expanding to reliably react in environments made challenging by adverse weather and/or traffic conditions. Certified autopilot systems, however, continue to presume a constant, known flight performance envelope, applying fixed guidance and control laws for each flight mode. When sustaining damage or experiencing failures, an aircraft's flight envelope will typically contract. Researchers have developed adaptive control laws to maintain stability and system identification methods to characterize degraded aircraft dynamics, but flight planning and guidance tasks still revert to the human pilot since fixed envelope assumptions no longer apply. In previous work, ${ }^{1-3}$ we developed an adaptive flight planning capability that automatically ranked and selected a nearby landing runway and built a trajectory to that runway under the assumption that either a Dubins path solution could be found or that sufficient flight planning time existed to identify a landing trajectory via search over sequences of feasible trim states. The search-based planner, however, often required nontrivial time (more than a minute in some cases) to identify a solution, during which time it was assumed the aircraft could remain stably aloft. This paper describes an extension to the Dubins path landing solution that enables a feasible landing trajectory to be generated even when straight flight is not possible. This solution requires only that the aircraft be capable of left or right turns of two different turning radii, enabling analytic construction of a sequence of spirals between initial state and the approach end of the landing runway. Scenarios in which the aircraft cannot maintain straight flight can result from a variety of conditions such as structural damage (e.g., to a wing) or actuator failures (e.g., stuck, fully-deflected rudder or ailerons).

Other researchers have begun to design flight management architectures that will assist the pilot in decision-making during emergencies. ${ }^{4}$ Researchers have also studied the aircraft trajectory planning problem for a variety of applications, including recent work on sequencing circular segments to allow a laser to consistently track a target. ${ }^{5}$ The classic engine-out (loss-of-thrust) scenario was addressed in our previous work by an extension of a Dubins path solver ${ }^{1}$ and has also been studied in the context of a turn-back landing cast in an optimal control framework. ${ }^{6}$ There also have been efforts to design multi-layer autonomous flight management systems for Unmanned Air Systems (UAS) such as the multi-layer intelligent control architecture. $^{7}$ We have previously modeled emergency situations ranging from loss of thrust ${ }^{1}$ to actuator failures $^{2}$ to a commercial transport with severe left wing damage. ${ }^{3}$ This work adopts the same framework as was introduced in our previous work, ${ }^{1}$ as shown in Figure 1. In the presence of failures and/or damage, the emergency flight planner activates the adaptive flight planner through a variable autonomy pilot interface and flight plan monitor. Within the AFP, a Landing Site Search (LSS) module determines a safe landing site, currently a runway deemed feasible based on length, width, wind conditions, etc. ${ }^{1}$ The Segmented Trajectory Planner constructs a sequence of valid post-failure trim states to this landing site. This paper presents an analytic trajectory planner that extends the analytic Dubins solver proposed for nominal or lossof-thrust scenarios thus reduces the requirement to use the computationally-intensive search-based solver. The remainder of this article is organized as follows. Section II describes the geometric constraints required to connect the initial turning flight segment with the final turn to touchdown. Section III presents the conditions under which the landing runway can be reached. Section IV describes the minimum-length trajectory plan for a case in which an aircraft experiences severe wing damage. Section V provides example landing trajectories, while section VI presents conclusions and future work.

\section{Sequence of Spirals Geometric Analysis}

To extend the Dubins path landing solution, we first define the concept of a Turning Dubins Vehicle.

Definition (Turning Dubins Vehicle (TDV)) A Turning Dubins Vehicle (TDV) is a planar vehicle that is constrained to move along paths of curvature bounded both above and below, without reversing direction and maintaining a constant speed.

Let $\sigma:[0, T] \rightarrow \mathbb{R}^{2}$ be a curve for the TDV that is twice differentiable for maneuver times $T>0$. For TDV

velocity $\vec{V}$ and unit tangent $\vec{T}=\frac{\vec{V}}{\|\vec{V}\|}$, the curvature vector $\vec{K}$ is defined as the rate of change of $\vec{T}$ with 


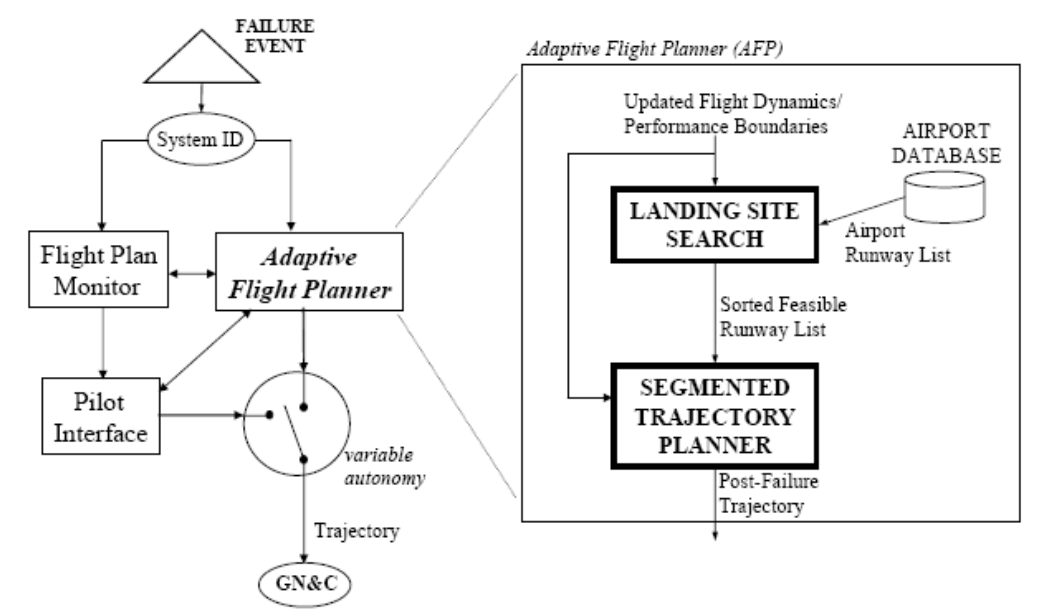

Figure 1. Emergency Flight Management Architecture.

respect to arc length $s$ :

$$
\begin{aligned}
\vec{K} & =\frac{d \vec{T}}{d s}=\frac{1}{\|\vec{V}\|} \dot{\vec{T}} \\
K & =\|\vec{K}\|=\frac{1}{r}
\end{aligned}
$$

where $r$ is the turning circle radius. Since $r_{m} \leq r \leq r_{M}$ where $r_{m}$ is the minimum turning radius and $r_{M}$ is the maximum turning radius, the magnitude of the curvature of $\sigma$ is bounded above by $\frac{1}{r_{m}}$ and bounded below by $\frac{1}{r_{M}}$. Let $\Sigma$ represent the set of possible curves for the TDV, i.e., $\Sigma=\left\{\sigma \mid K \in\left[\frac{1}{r_{M}}, \frac{1}{r_{m}}\right]\right\}$.

Since our landing solution requires only left or right turns of two different radii, we shall use $\mathcal{O}$ to denote a circle. Given a center $c$ in $\mathbb{R}^{2}$, a radius $r$, and a sign of the turning rate $\operatorname{sgn}(\dot{\psi})$, let $\mathcal{O}(c, r, \operatorname{sgn}(\dot{\psi}))$ : $\left[0, T_{\mathcal{O}}\right] \rightarrow \mathbb{R}^{2}$ represent a circle of radius $r$ with center $c$ and direction of motion $\operatorname{sgn}(\dot{\psi})$ where $T_{\mathcal{O}}$ denotes the maneuver time during $\mathcal{O}$ and let $\Sigma_{c}$ be the set of circular curves for the TDV as follows:

$$
\Sigma_{c}=\left\{\mathcal{O}(c, r, \operatorname{sgn}(\dot{\psi})) \mid r_{m} \leq r \leq r_{M}, \operatorname{sgn}(\dot{\psi})=\left\{\begin{array}{ll}
+ & \text { if } \dot{\psi}>0 \\
- & \text { if } \dot{\psi}<0
\end{array}\right\}\right.
$$

To obtain the landing trajectory, we identify a reference arc that can be followed by alternating segments of two different turning radii that include a predefined safety factor sufficient for disturbance rejection. For a given center $c$ in $\mathbb{R}^{2}$ and two given points $p_{i}$ and $p_{f}$ in $\mathbb{R}^{2}$, let $a\left(c, p_{i}, p_{f}\right):\left[0, T_{a}\right] \rightarrow \mathbb{R}^{2}$ be a circular arc connecting $p_{i}$ and $p_{f}$ with arc center $c$ and let $\mathcal{A}=\left\{a\left(c, p_{i}, p_{f}\right) \mid c, p_{i}, p_{f} \in \mathbb{R}^{2}\right\}$. As shown in Figure $2, \mathcal{O}_{i}$ and $\mathcal{O}_{f}$ in $\Sigma_{c}$ represent the initial and final circular curves, respectively, and would formerly have represented the initial and final arcs from which a connecting (straight) tangent would have been computed for a Dubins path solution. $\mathcal{O}_{f}$ is tangent to an extension line from the runway, guiding the aircraft to the desired touchdown state at the landing runway's approach end. The curvature vector $\vec{K}$ is orthogonal to $\vec{T}$ when $\vec{T}$ is on the circle. Let $\vec{r}_{E}=\frac{1}{2}\left(\vec{r}_{c_{i}}+\vec{r}_{c_{f}}\right)$. As shown in Figure 2, since $\triangle O c_{i} E$ and $\triangle O c_{f} E$ are congruent, the centers of the reference arc $a_{r}$ lie on a straight line passing through $\mathrm{E}$ and $\mathrm{F}$. In order to find a vector $\vec{K}_{O}$ that 


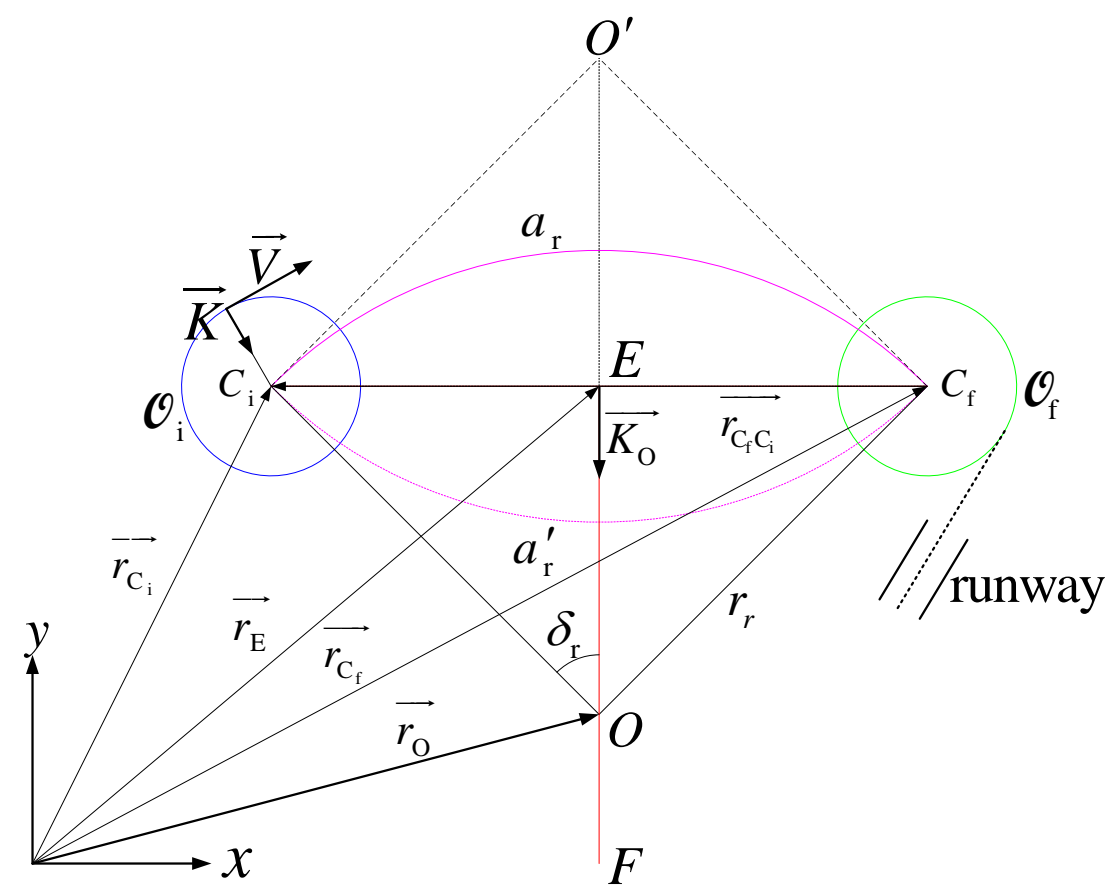

Figure 2. Arcs Connecting Initial and Final Approach Turns

represents straight line $\overline{E F}$ perpendicular to $\vec{r}_{c_{f} c_{i}}$, we represent curvature vector $\vec{K}$ in polar coordinates :

$$
\vec{K}_{i}=\left[\begin{array}{c}
K \cos \zeta_{i} \\
K \sin \zeta_{i}
\end{array}\right]
$$

Let $\vec{r}_{c_{f} c_{i}}=\left[\begin{array}{c}x_{c_{f} c_{i}} \\ y_{c_{f} c_{i}}\end{array}\right]$. Since $\vec{K}_{O}$ is perpendicular to $\vec{r}_{c_{f} c_{i}}$,

$$
\begin{gathered}
\vec{K}_{O} \cdot \vec{r}_{c_{f} c_{i}}=x_{c_{f} c_{i}} K \cos \zeta_{O}+y_{c_{f} c_{i}} K \sin \zeta_{O}=0 \\
\zeta_{O}=\arctan \left(-\frac{x_{c_{f} c_{i}}}{y_{c_{f} c_{i}}}\right)
\end{gathered}
$$

Hence, $\vec{r}_{O}$ to the center $O$ of the arc is given by

$$
\vec{r}_{O}=\vec{r}_{E}+\lambda \vec{K}_{O}
$$

where $\lambda$ has a range from $-\infty$ to $\infty$ and $\vec{K}_{O}=\left[\begin{array}{c}K \cos \zeta_{O} \\ K \sin \zeta_{O}\end{array}\right]$. Furthermore, since $\triangle O c_{f} c_{i}$ and $\triangle O^{\prime} c_{f} c_{i}$ are congruent, there also exists a dual reference arc, $a_{r}^{\prime}$, as shown in Figure 2. Hence, we can choose one of the two arcs according to travel direction $\operatorname{sgn}(\dot{\psi})$ around the turning circle. Let $\mathcal{A}_{r}$ be the set of possible reference arcs connecting centers $c_{i}$ and $c_{f}$ as follows :

$$
\mathcal{A}_{r}=\left\{a\left(O, c_{i}, c_{f}\right) \mid \vec{r}_{O}=\frac{1}{2}\left(\vec{r}_{c_{i}}+\vec{r}_{c_{f}}\right)+\lambda \vec{K}_{O}, \quad-\infty \leq \lambda \leq \infty\right\}
$$


By using $\vec{r}_{O c_{i}}$, we can calculate the radius $r_{r}$, angle $\delta_{r}$, and length $l_{r}$ of the reference arc $a_{r} \in \mathcal{A}_{r}$, as shown in Figure 2:

$$
\begin{aligned}
& r_{r}=\left\|\vec{r}_{O c_{i}}\right\| \\
& \delta_{r}=\left\{\begin{array}{lll}
\arccos \left(\frac{\vec{r}_{O c_{i}} \cdot\left(\vec{r}_{E}-\vec{r}_{O}\right)}{\left\|\vec{r}_{O c_{i}}\right\|\left\|\vec{r}_{E}-\vec{r}_{O}\right\|}\right) & \text { if } & 0 \leq \lambda \leq \infty \\
\pi-\arccos \left(\frac{\vec{r}_{O c_{i}} \cdot\left(\vec{r}_{E}-\vec{r}_{O}\right)}{\left\|\vec{r}_{O c_{i}}\right\|\left\|\vec{r}_{E}-\vec{r}_{O}\right\|}\right) & \text { if } & -\infty \leq \lambda<0
\end{array}\right. \\
& l_{r}=2 r_{r} \delta_{r}
\end{aligned}
$$

If $a_{2 i-1}\left(T_{2 i-1}\right)=a_{2 i}(0)$ where $a_{2 i-1}$ and $a_{2 i}$ are the $\operatorname{arcs}$ of $\mathcal{O} \in \Sigma_{c}$ intercepted by $a_{r} \in \mathcal{A}_{r}$, then we define a product of two arcs as :

$$
b_{i}=a_{2 i-1} * a_{2 i}= \begin{cases}a_{2 i-1}\left(t-T_{2 i-2}\right), & T_{2 i-2} \leq t \leq T_{2 i-1} \\ a_{2 i}\left(t-T_{2 i-1}\right), & T_{2 i_{1}} \leq t \leq T_{2 i}\end{cases}
$$

where $T_{0}=0$.

Theorem II.1 Let $\mathcal{O}_{1}\left(c_{1}, r_{1}, \operatorname{sgn}(\dot{\psi})\right)$ and $\mathcal{O}_{2}\left(c_{2}, r_{2}\right.$, sgn $\left.(\dot{\psi})\right)$ be in $\Sigma_{c}$ with $r_{1} \neq r_{2}$. Let $a_{1}$ be the intercepted arc of $\mathcal{O}_{1}$ with $a_{r} \in \mathcal{A}_{r}$ and center $c_{1}$ such that $a_{1}\left(c_{1}, p_{0}, p_{1}\right):\left[0, T_{1}\right] \rightarrow \mathbb{R}^{2}$ where $p_{0}$ and $p_{1}$ lie on $a_{r}$. Let $a_{2}$ be the intercepted arc of $\mathcal{O}_{2}$ with $a_{r} \in \mathcal{A}_{r}$ and center $c_{2}$. Suppose $c_{1}$ lies on arc $a_{r}$. If $c_{2}$ is located on straight line $\overline{p_{1} c_{1}}$ with distance $r_{2}$ from $p_{1}$, then $\mathcal{O}_{1}$ and $\mathcal{O}_{2}$ are tangent. Moreover, the change in heading angle of a TDV over $a_{1} * a_{2}$ is either $2 \pi+4 \delta_{1}-2 \delta_{2}$ or $2 \pi-4 \delta_{1}+2 \delta_{2}$ according to sgn $(\dot{\psi})$ where $\delta_{1}=\arcsin \left(\frac{r_{1}}{2 r_{r}}\right)$ and $\delta_{2}=\arccos \left(\frac{\vec{a}_{O c_{2}} \cdot \vec{a}_{O p_{1}}}{\left\|\vec{a}_{O c_{2}}\right\| r_{r}}\right)$.

Proof Assume $c_{2}$ is on straight line $\overline{p_{1} c_{1}}$ with distance $r_{2}$ from $p_{1}$. Then, the TDV velocity, $\vec{V}$, is perpendicular to $\vec{r}_{p_{1} c_{1}}$ at $p_{1}$. Since $c_{2}$ is located on the straight line $\overline{p_{1} c_{1}}, \vec{r}_{p_{1} c_{1}}$ and $\vec{r}_{p_{1} c_{2}}$ are parallel, and $\vec{V}$ is perpendicular to $\vec{r}_{p_{1} c_{2}}$ at $p_{1}$. Hence, $\mathcal{O}_{1}$ and $\mathcal{O}_{2}$ are tangent.

Since $\mathcal{O}_{1}$ and $\mathcal{O}_{2}$ are tangent at $p_{1}, a_{1}\left(T_{1}\right)=a_{2}(0)=p_{1}$, i.e. $a_{2}\left(c_{2}, p_{1}, p_{2}\right):\left[0, T_{2}\right] \rightarrow \mathbb{R}^{2}$ where $p_{1}$ and $p_{2}$ lie on $a_{r}$. Hence, the product of two $\operatorname{arcs} a_{1} * a_{2}$ is defined. Given the assumption $r_{1} \neq r_{2}, r_{1}>r_{2}$ or $r_{1}<r_{2}$, as shown in Figure 3 for $i=1$. For both cases, $\triangle O c_{2} p_{1}$ and $\triangle O c_{2} p_{2}$ are congruent where $p_{2}$ is another intersection point of $\mathcal{O}_{2}$ and $a_{r} \in \mathcal{A}_{r}$. Since $\angle O p_{1} c_{1}$ is $\frac{\pi}{2}-\delta_{1}$, the central angles of $\mathcal{O}_{2}$ including $\tau_{2}$ and $\rho_{2}$ are given by :

$$
\begin{aligned}
\tau_{2} & =\pi+2\left(\delta_{1}-\delta_{2}\right) \\
\rho_{2} & =2 \pi-\tau_{2}
\end{aligned}
$$

Since $\triangle O p_{1} c_{1}$ is an isosceles triangle, the central angles of $\mathcal{O}_{1}$ including $\tau_{1}$ and $\rho_{1}$ are given by :

$$
\begin{aligned}
& \tau_{1}=\pi-2 \delta_{1} \\
& \rho_{1}=\pi+2 \delta_{1}
\end{aligned}
$$

We now determine angles $\delta_{1}$ and $\delta_{2}$. Since $\triangle O p_{1} c_{1}$ is an isosceles triangle,

$$
\delta_{1}=\arcsin \left(\frac{r_{1} / 2}{r_{r}}\right)
$$

Note that the length of the arc $a_{r}$ intercepted by $\mathcal{O}_{1}, l_{1}$, is $4 r_{r} \delta_{1}$. To compute $\delta_{2}$, define $\vec{r}_{O p_{1}}$ as a vector from $\vec{r}_{O c_{1}}$ rotated $-4 \delta_{1}$ about the $\mathrm{z}$ axis:

$$
\vec{r}_{O p_{1}}=\left[\begin{array}{cc}
\cos \left(-4 \delta_{1}\right) & -\sin \left(-4 \delta_{1}\right) \\
\sin \left(-4 \delta_{1}\right) & \cos \left(-4 \delta_{1}\right)
\end{array}\right] \vec{r}_{O c_{1}}
$$

Since $\vec{r}_{p_{1} c_{2}}=\frac{r_{2}}{r_{1}} \vec{p}_{p_{1} c_{1}}$ and $\vec{r}_{O c_{2}}=\vec{r}_{O p_{1}}+\vec{r}_{p_{1} c_{2}}$,

$$
\therefore \delta_{2}=\arccos \left(\frac{\vec{r}_{O c_{2}} \cdot \vec{r}_{O p_{1}}}{\left\|\vec{r}_{O c_{2}}\right\| r_{r}}\right)
$$


Note that the length of the arc of $a_{r}$ intercepted by $\mathcal{O}_{2}, l_{2}$, is $2 r_{r} \delta_{2}$. Since the TDV doesn't reverse direction, we only consider two permutations about central angles of $\mathcal{O}_{1}$ and $\mathcal{O}_{2}$ according to $\operatorname{sgn}(\dot{\psi})$ :

$$
\left\{\rho_{1}, \tau_{2}\right\} \quad \text { or } \quad\left\{\tau_{1}, \rho_{2}\right\}
$$

Hence, the change in heading angle of the TDV in $a_{1} * a_{2}$ is given by:

$$
\begin{array}{lll}
2 \pi+4 \delta_{1}-2 \delta_{2} & \text { for } & \left\{\rho_{1}, \tau_{2}\right\} \\
2 \pi-4 \delta_{1}+2 \delta_{2} & \text { for } & \left\{\tau_{1}, \rho_{2}\right\}
\end{array}
$$

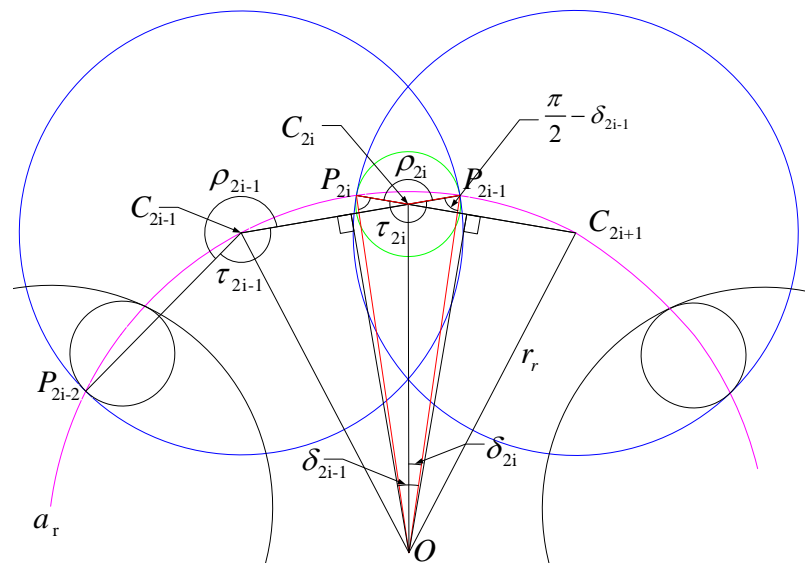

(a) $r_{2 i-1}>r_{2 i}$

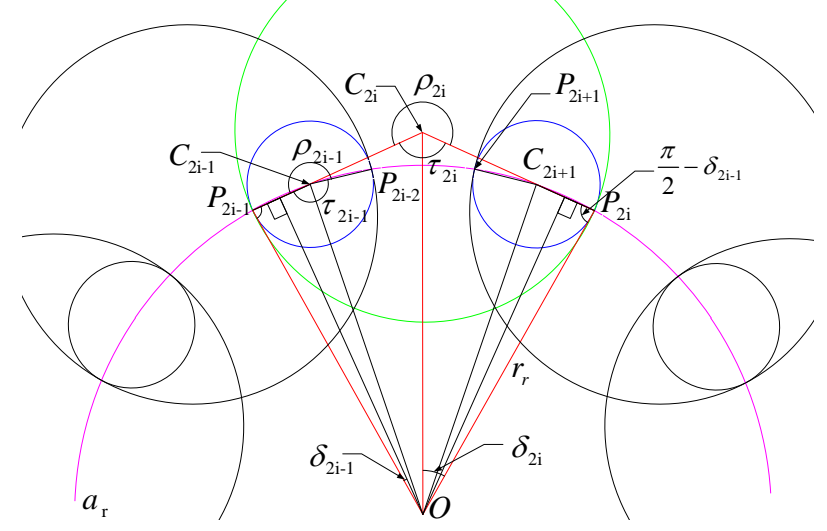

(b) $r_{2 i-1}<r_{2 i}$

Figure 3. Product of $\mathbf{n}$ arcs

Corollary II.2 Let $\mathcal{O}_{2 i-1}\left(c_{2 i-1}, r_{2 i-1}, \operatorname{sgn}(\dot{\psi})\right)$ and $\mathcal{O}_{2 i}\left(c_{2 i}, r_{2 i}, \operatorname{sgn}(\dot{\psi})\right)$ be in $\Sigma_{c}$ with $r_{2 i-1} \neq r_{2 i}$ and $r_{i}=r_{i+2}$ for all $i \in \mathbb{N}$. Let $a_{1}$ be the intercepted arc of $\mathcal{O}_{1}$ with $a_{r} \in \mathcal{A}_{r}$ and center $c_{1}$ such that $a_{1}\left(c_{1}, p_{0}, p_{1}\right):\left[0, T_{1}\right] \rightarrow \mathbb{R}^{2}$ where $p_{0}$ and $p_{1}$ lie on $a_{r}$. Suppose $c_{1}$ lies on $a_{r}$. If $c_{2 i}$ is located on straight line $\overline{p_{2 i-1} c_{2 i-1}}$ with distance $r_{2}$ from $p_{2 i-1}$ and $c_{2 i+1}$ is located on straight line $\overline{p_{2 i} c_{2 i}}$ with distance $r_{1}$ from $p_{2 i}$ for all $i \in \mathbb{N}$ where $p_{2 i-1}$ and $p_{2 i}$ lie on $a_{r}$, then $\mathcal{O}_{2 i-1}$ and $\mathcal{O}_{2 i}$ are tangent at $p_{2 i-1}$, and $\mathcal{O}_{2 i}$ and $\mathcal{O}_{2(i+1)-1}$ at $p_{2 i}$ exist such that $c_{2 i-1}$ and $c_{2(i+1)-1}$ lie on $a_{r}$ for all $i \in \mathbb{N}$. Moreover, the change in heading angle of the TDV over $\left\{b_{i} \mid i \in\{1,2, \cdots, n\}\right\}$ is either $n\left(2 \pi+4 \delta_{1}-2 \delta_{2}\right)$ or $n\left(2 \pi-4 \delta_{1}+2 \delta_{2}\right)$ where $\delta_{1}=\arcsin \left(\frac{r_{1} / 2}{r_{r}}\right)$ and $\delta_{2}=\arccos \left(\frac{\vec{a}_{O c_{2}} \cdot \vec{a}_{O p_{1}}}{\left\|\vec{a}_{O c_{2}}\right\| r_{r}}\right)$.

Proof Suppose $c_{2 i}$ is located on straight line $\overline{p_{2 i-1} c_{2 i-1}}$ with distance $r_{2}$ from $p_{2 i-1}$ and $c_{2 i+1}$ is located on straight line $\overline{p_{2 i} c_{2 i}}$ with distance $r_{1}$ from $p_{2 i}$ where $p_{2 i-1}$ and $p_{2 i}$ lie on $a_{r}$ for all $i \in \mathbb{N}$. Since $c_{1}$ lies on $a_{r}, \mathcal{O}_{1}$ and $\mathcal{O}_{2}$ are tangent at $p_{1}$ and the product of two $\operatorname{arcs} a_{1} * a_{2}$ is defined such that $a\left(c_{2}, p_{1}, p_{2}\right):\left[0, T_{2}\right] \rightarrow \mathbb{R}^{2}$ where $p_{1}$ and $p_{2}$ lie on $a_{r}$ from Theorem II.1. Since the perperndicular bisector of a chord contains the center of the circle, $\triangle O c_{2} p_{1}$ and $\triangle O c_{2} p_{2}$ are congurent, and $\angle O p_{2} c_{2}=\angle O p_{1} c_{2}$. By triangle congruence, $\overline{O c_{3}}=r_{r}$, and $c_{3}$ lies on $a_{r}$. Since the velocity of the TDV, $\vec{V}$, is perpendicular to $\vec{r}_{p_{2} c_{2}}$ and $\vec{r}_{p_{2} c_{3}}$ at $p_{2}, \mathcal{O}_{2}$ and $\mathcal{O}_{3}$ are tangent.

Let the nth proposition be that $\mathcal{O}_{2 n-1}$ and $\mathcal{O}_{2 n}$ are tangent at $p_{2 n-1}$, and $\mathcal{O}_{2 n}$ and $\mathcal{O}_{2 n+1}$ at $p_{2 n}$ exist such that $c_{2 n-1}$ and $c_{2 n+1}$ lie on $a_{r}$. Suppose our nth proposition is true. From Theorem II.1, $\mathcal{O}_{2 n+1}$ and $\mathcal{O}_{2 n+2}$ are tangent at $p_{2 n+1}$ because $c_{2 n+1}$ lies on $a_{r}$. Since $\triangle O p_{2 n+1} c_{2 n+1}$ and $\triangle O p_{2 n+2} c_{2 n+3}$ are congruent, $c_{2 n+3}$ lies on $a_{r}$, and $\mathcal{O}_{2 n+2}$ and $\mathcal{O}_{2 n+3}$ are tangent at $p_{2 n+2}$.

Since $\mathcal{O}_{2 i-1}$ and $\mathcal{O}_{2 i}$ are tangent at $p_{2 i-1}$ for all $i \in \mathbb{N}, a_{2 i-1}\left(T_{2 i-1}\right)=a_{2 i}(0)=p_{2 i-1}$, and $b_{i}$ is defined for all $i \in \mathbb{N}$. If $b_{i}\left(T_{2} i\right)=b_{i+1}(0)$ where $b_{i}=a_{2 i-1} * a_{2 i}$ and $b_{i+1}=a_{2(i+1)-1} * a_{2(i+1)}$, then we define a product of two products as :

$$
b_{i} * b_{i+1}= \begin{cases}b_{i}\left(t-T_{2 i-2}\right), & T_{2 i-2} \leq t \leq T_{2 i} \\ b_{i+1}\left(t-T_{2 i}\right), & T_{2 i} \leq t \leq T_{2 i+2}\end{cases}
$$


where $T_{0}=0$. Since $\mathcal{O}_{2 i}$ and $\mathcal{O}_{2(i+1)-1}$ are tangent at $p_{2 i}$ for all $i \in \mathbb{N}, b_{1} * b_{2} * \cdots * b_{n}$ is defined for all $i \in \mathbb{N}$. Let $\mathcal{B}$ represent the set of possible sequences of two different turning radii for the TDV in $a_{r} \in \mathcal{A}_{r}$, i.e. $\mathcal{B}=\left\{b_{i} \mid i \in\{1,2, \cdots, n\}\right\}$ over $a_{r}$.

For $n=1$, it is true from Theorem II.1 that the change in heading angle of the TDV in $b_{1}$ is either $2 \pi-4 \delta_{1}-2 \delta_{2}$ or $2 \pi-4 \delta_{1}+2 \delta_{2}$ according to $\operatorname{sgn}(\dot{\psi})$. Suppose our nth proposition is true. Since $\mathcal{O}_{2 n}$ and $\mathcal{O}_{2(n+1)-1}$ are tangent at $p_{2 n}$ and $c_{2(n+1)-1}$ lies on $a_{r}$, the change in heading angle of the TDV in $b_{n+1}$ defined by $\mathcal{O}_{2(n+1)-1}$ and $\mathcal{O}_{2(n+1)}$ is $2 \pi+4 \delta_{1}-2 \delta_{2}$ or $2 \pi-4 \delta_{1}+2 \delta_{2}$ according to $\operatorname{sgn}(\dot{\psi})$. Hence, the $(n+1)$ th proposition is true. By induction, the change in heading angle of the TDV in $\left\{b_{i} \mid i \in\{1,2, \cdots, n\}\right\}$ for all $n \in \mathbb{N}$ is either $n\left(2 \pi+4 \delta_{1}-2 \delta_{2}\right)$ or $n\left(2 \pi-4 \delta_{1}+2 \delta_{2}\right)$ according to $\operatorname{sgn}(\dot{\psi})$.

\section{Landing Trajectory Feasibility Condition}

In this section, we present criteria by which a particular landing site is feasible(reachable) with a TDV trajectory about reference arc $a_{r}$. From Corollary II.2, $\mathcal{B}=\left\{b_{i} \mid i \in\{1,2, \cdots, n\}\right\}$ over $a_{r} \in \mathcal{A}_{r}$. After $n$ sequences, however, $\mathcal{O}_{f}$ and $\mathcal{O}_{2 n}$ are not guaranteed tangent. The following theorem describes the feasibility condition about $a_{r}$ for the TDV to reach the selected runway.

Theorem III.1 Consider $\mathcal{B}=\left\{b_{i} \mid i \in\{1,2, \cdots, n\}\right\}$ over $a_{r} \in \mathcal{A}_{r}$. Let $\mathcal{O}_{1}$ and $\mathcal{O}_{f}$ represent the initial and final circular curves, respectively. Then there exists an $a_{r}$ such that $\mathcal{O}_{f}$ and $\mathcal{O}_{2 n}$ are tangent at $p_{2 n}$ if and only if $r_{r}$ satisfies the feasibility condition:

$$
\delta_{r}-n\left|2 \delta_{1}-\delta_{2}\right|=0
$$

where $\delta_{r}=\left\{\begin{array}{ll}\arcsin \left(\frac{\left\|\vec{r}_{C_{f} c_{i}}\right\|}{2 r_{r}}\right) & \text { if } 0 \leq \lambda \leq \infty \\ \pi-\arcsin \left(\frac{\left\|\vec{r}_{C_{f}} c_{i}\right\|}{2 r_{r}}\right) & \text { if }-\infty \leq \lambda<0\end{array}, \delta_{1}=\arcsin \left(\frac{r_{1}}{2 r_{r}}\right)\right.$, and $\delta_{2}=\arctan \left(\frac{\sqrt{r_{r}^{2}-\frac{r_{1}^{2}}{4}}}{\frac{r_{r}^{2}}{r_{2}}-\frac{r_{1}}{2}}\right)$.

Hence, the TDV can reach the selected runway.

Proof Suppose there exists an $a_{r}$ such that $\mathcal{O}_{f}$ and $\mathcal{O}_{2 n}$ are tangent at $p_{2 n}$. Hence, $\mathcal{O}_{f}$ is the additional circular curve, i.e., $\mathcal{O}_{2 n+1}$, such that $\mathcal{O}_{2 n}$ and $\mathcal{O}_{2 n+1}$ are tangent at $p_{2 n}$. Considering the imaginary $\mathcal{O}_{2 n+2}$ such that $\mathcal{O}_{2 n+1}$ and $\mathcal{O}_{2 n+2}$ are tangent, we can define an additional segment $b_{n+1} \in\left\{b_{i} \mid i \in \mathbb{N}\right\}$ over $a_{r}$. From Corollary II.2, the change in heading angle of the TDV over each $b_{i}$ is identical except case $b_{1}$, and the length of $a_{r} \in \mathcal{A}_{r}$ traversed over each $b_{i}$ is also identical except case $b_{1}$. Note that the change in heading angle of the TDV over $b_{1}$ is different because of the TDV's initial position, $p_{0}$.

Let $J$ represent the length of $a_{r} \in \mathcal{A}_{r}$ traversed over each $b_{i}$ for all $i \in\{2,3, \cdots, n\}$ and $l_{J}$ represent the length of $a_{r}$ traversed over $\left\{b_{i} \mid i \in\{2,3, \cdots, n\}\right\}$. Since $J$ is independent of $i \in\{2,3, \cdots, n\}$,

$$
l_{J}=(n-1) J
$$

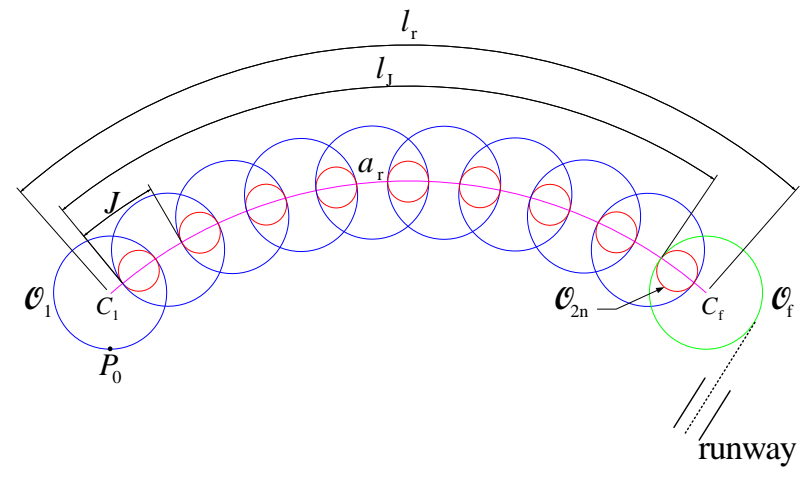

(a) $r_{1}>r_{2}$

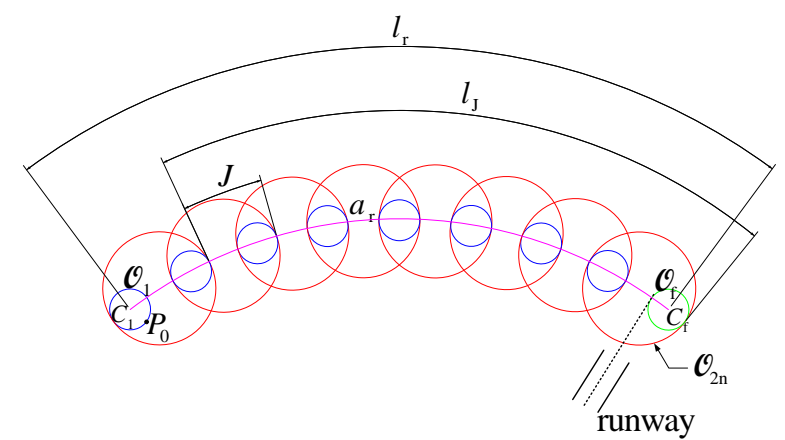

(b) $r_{1}<r_{2}$

Figure 4. Feasibility Condition 
where $n \in \mathbb{N}$. Hence, $l_{J}$ must be an integer multiple of $J$ for the runway to be reached via $\left\{b_{i} \mid i \in\{1,2, \cdots, n\}\right\}$. From Theorem II.1, $l_{1}$ and $l_{2}$ represent the length of $a_{r} \in \mathcal{A}_{r}$ traversed over each $a_{2 i-1}$ and $a_{2 i}$, respectively, such that $b_{i}=a_{2 i-1} * a_{2 i}$ for all $i \in\{2,3, \cdots, n\}$, and are defined as :

$$
l_{1}=4 r_{r} \delta_{1} \quad \text { and } \quad l_{2}=2 r_{r} \delta_{2}
$$

From Eq. 25, $l_{J}=l_{r}-\left|l_{1}-l_{2}\right|$, and $J=\left|l_{1}-l_{2}\right|$. Substituting Eqs. 11 and 26, the feasibility condition for $a_{r} \in \mathcal{A}_{r}$ can be determined:

$$
\delta_{r}-n\left|2 \delta_{1}-\delta_{2}\right|=0
$$

where $\delta_{r}=\left\{\begin{array}{ll}\arcsin \left(\frac{\left\|\vec{r}_{C_{f} c_{i}}\right\|}{2 r_{r}}\right) & \text { if } \quad 0 \leq \lambda \leq \infty \\ \pi-\arcsin \left(\frac{\left\|\vec{r}_{C_{f}} c_{i}\right\|}{2 r_{r}}\right) & \text { if } \quad-\infty \leq \lambda<0\end{array}, \delta_{1}=\arcsin \left(\frac{r_{1}}{2 r_{r}}\right)\right.$, and $\delta_{2}=\arctan \left(\frac{\sqrt{r_{r}^{2}-\frac{r_{1}^{2}}{4}}}{\frac{r_{r}^{2}}{r_{2}}-\frac{r_{1}}{2}}\right)$. Note that $l_{1}>l_{2}$ if $r_{1}>r_{2}$ and $l_{1}<l_{2}$ if $r_{1}<r_{2}$, and $r_{2 i-1} \neq r_{2 i}$ and $r_{i}=r_{i+2}$ in $\mathcal{O}_{2 i-1}\left(c_{2 i-1}, r_{2 i-1}, \operatorname{sgn}(\dot{\psi})\right)$ and $\mathcal{O}_{2 i}\left(c_{2 i}, r_{2 i}, \operatorname{sgn}(\dot{\psi})\right)$ for all $i \in \mathbb{N}$. Since $\mathcal{O}_{f}$ and $\mathcal{O}_{2 n}$ are tangent at $p_{2 n}$, the TDV can reach the selected runway. Suppose $r_{r}$ satisfies the feasibility condition in Eq. 24.

$$
\therefore l_{J}=(n-1) J
$$

Since the length of $a_{r} \in \mathcal{A}_{r}$ traversed over each $b_{i}$ is identical except the case in $b_{1}$, we take $a_{r}$ such that $l_{J}=(n-1) J$. Since $l_{J}$ is the length of $a_{r}$ from $p_{2}$ to $p_{2 n}$ from the definition, $\mathcal{O}_{2 n}$ and $\mathcal{O}_{2 n+1}$ are tangent at $p_{2 n}$, and $c_{2 n+1}$ lies on $a_{r}$ by Corollary II.2. When $r_{1}>r_{2}$, the sum of the length of $a_{r}$ from $c_{1}$ to $p_{2}$ and the length of $a_{r}$ from $p_{2 n}$ to $c_{2 n+1}$ is $J$, and the length of $a_{r}$ from $c_{1}$ to $c_{2 n+1}$ is $l_{r}$. Moreover, when $r_{1}<r_{2}$, the difference of the length of $a_{r}$ from $c_{1}$ to $p_{2}$ and the length of $a_{r}$ from $c_{2 n+1}$ to $p_{2 n}$ is $J$, and the length of $a_{r}$ from $c_{1}$ to $c_{2 n+1}$ is $l_{r}$. Since $l_{r}$ is the length of $a_{r}$ from $c_{1}$ to $c_{f}$ from the definition, $c_{2 n+1}$ is $c_{f}$, and $c_{2 n}$ and $c_{f}$ are tangent at $p_{2 n}$. Hence, the selected runway can be reached. Note that if $r_{1}<r_{2}, p_{1}$ is located on the extension arc from $c_{1}$ in $a_{1}\left(c_{1}, p_{0}, p_{1}\right)$.

In Figure 5, we specify a waypoint generation algorithm (WGA) aimed at a practical, real-time solution that extends our previous Dubins path strategy ${ }^{1}$ to cases in which straight flight is not feasible. If straight flight is possible, the existing segmented trajectory planner constructs a Dubins path of guaranteed minimum length to the top-ranked landing runway. ${ }^{1}$ Otherwise, we adopt the lateral plane trajectory of alternatingradius turns described in this paper, with flight path angle set to a value that ensures touchdown at the runway altitude. ${ }^{a}$ We define the initial and final turning circle centers, from which the set of feasible reference arcs can be determined as described below.

\section{Analytic Determination of the Minimum Turning Sequence}

Since $\lambda$ has range $-\infty$ to $\infty$ in $\mathcal{A}_{r}$, we can find a lower bound for $n$ in $\left\{b_{i} \mid i \in\{1,2, \cdots, n\}\right\}$ as shown in Figure 6. $\mathcal{A}_{r}$ evolves from a straight line to a circle as $\lambda$ is varied from $\infty$ to $-\infty$. Moreover, since our landing solution requires only left or right turns of two different radii and $r_{m} \leq r \leq r_{M}, J$ is bounded. Let $n_{m}$ represent the minimum value of $n$ such that $J$ has the maximum value satisfying the feasibility condition, and let $a_{r_{m}}$ represent the reference arc having $n_{m}$.

Theorem IV.1 Consider $\mathcal{B}=\left\{b_{i} \mid i \in\{1,2, \cdots, n\}\right\}$ over $a_{r} \in \mathcal{A}_{r}$. Let $\mathcal{O}_{1}$ and $\mathcal{O}_{f}$ represent the initial and final circular curves, respectively. If $l_{r}=n J$, then $n_{m}=\left\lceil\frac{\left\|\vec{r}_{c_{f} c_{i}}\right\|}{\left|2 r_{1}-2 r_{2}\right|}\right\rceil$ and there exists $a_{r_{m}}$ such that $\mathcal{O}_{2 n}$ and $\mathcal{O}_{f}$ are tangent at $p_{2 n}$ where

$$
\begin{array}{lllll}
r_{1}=r_{M} & \text { and } & r_{2}=r_{m} & \text { if } & r_{1}>r_{2} \\
r_{1}=r_{m} & \text { and } & r_{2}=r_{M} & \text { if } & r_{1}<r_{2}
\end{array}
$$

Proof Suppose $l_{r}=n J=n\left|l_{1}-l_{2}\right|$. From the definition of $\mathcal{A}_{r}, \mathcal{A}_{r}$ evolves from a straight line to a circle as $\lambda$ is varied from $\infty$ to $-\infty$. Hence, $\left\|\vec{r}_{c_{f} c_{i}}\right\| \leq l_{r}$.

$$
\frac{\left\|\vec{r}_{c_{f} c_{i}}\right\|}{\left|l_{1}-l_{2}\right|} \leq n
$$

${ }^{a}$ For simplicity we currently presume the damage/failure does not compromise the aircraft's ability to command nominal descending flight path angles $\gamma$ for the sequenced turning segments. 


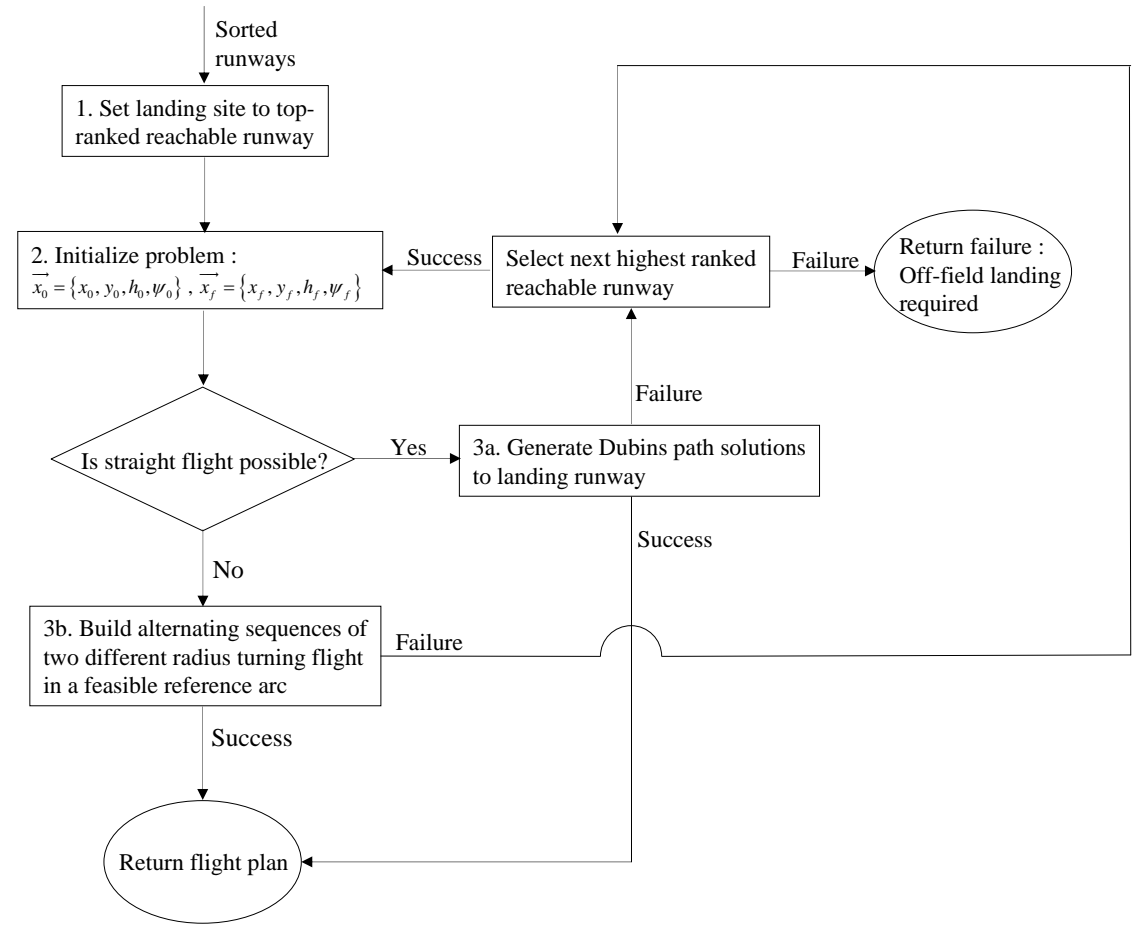

Figure 5. Waypoint Generation Algorithm (WGA)

Since $r_{m} \leq r \leq r_{M}$, we can find bounds for $\left|l_{1}-l_{2}\right|$. The following lemma bounds this maximum value of $J$.

Lemma IV.2 If circular curves of two distinct radii satisfy the condition:

$$
\begin{array}{lllll}
r_{1}=r_{M} & \text { and } & r_{2}=r_{m} & \text { if } & r_{1}>r_{2} \\
r_{1}=r_{m} & \text { and } & r_{2}=r_{M} & \text { if } & r_{1}<r_{2}
\end{array}
$$

then $J$ has the maximum value for all $r_{r}$.

Proof Let $r_{r} \in \mathbb{R}$ and $r_{r} \geq \frac{\left\|\vec{r}_{c_{f} c_{i}}\right\|}{2}$. To prove the Lemma, we compute the maximum value of $\left|2 \delta_{1}-\delta_{2}\right|$ for all $r_{r}$, and we maximize the difference between $2 \delta_{1}$ and $\delta_{2}$. First, consider the case where $r_{1}>r_{2}$. Without loss of generality, let $r_{1}=r_{M}$. As $r_{2}$ is varied from $r_{m}$ to $r_{M}, c_{2}$ converges to $c_{1}$ along the line $\overline{p_{1} c_{1}}$ as shown in Figure 2 for $i=1$. Hence, $\delta_{2}$ is given by:

$$
\begin{aligned}
& \delta_{2}=\delta_{1}-\arctan \left(\frac{\frac{r_{M}}{2}-r_{2}}{\sqrt{r_{r}^{2}-\frac{r_{M}^{2}}{4}}}\right) \quad \text { if } \quad r_{m} \leq r_{2} \leq \frac{r_{M}}{2} \\
& \delta_{2}=\delta_{1}+\arctan \left(\frac{r_{2}-\frac{r_{M}}{2}}{\sqrt{r_{r}^{2}-\frac{r_{M}^{2}}{4}}}\right) \quad \text { if } \quad \frac{r_{M}}{2} \leq r_{2} \leq r_{M}
\end{aligned}
$$

If $\frac{r_{M}}{2} \leq r_{m}$, then we only consider Eq. 35. Since the arc tangent in Eqs. 34 and 35 is nondecreasing for the tangent angle ranging $-\frac{\pi}{2}$ to $\frac{\pi}{2}, \delta_{2}$ has the minimum value if $r_{2}=r_{m}$. Furthermore, since $\delta_{1}$ is independent of $r_{2}, \delta_{1}$ has the maximum value if $r_{1}=r_{M}$. For $r_{1}>r_{2}$, if $r_{1}=r_{M}$ and $r_{2}=r_{m}$, then $J$ has the maximum value for all $r_{r}$. Note that since we consider all $r_{r}, r_{r}$ depends on the two centers of the initial and final circular curves. Next, we consider the case where $r_{1}<r_{2}$. The second case is the same as the first case setting $r_{1}=r_{m}$ and $r_{2}=r_{M}$. Note that for the second case, $\delta_{2}$ is given by:

$$
\delta_{2}=\delta_{1}+\arctan \left(\frac{r_{2}-\frac{r_{m}}{2}}{\sqrt{r_{r}^{2}-\frac{r_{m}^{2}}{4}}}\right)
$$




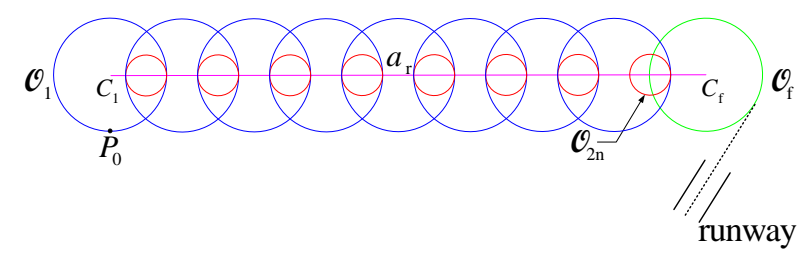

(a) $r_{1}>r_{2}$

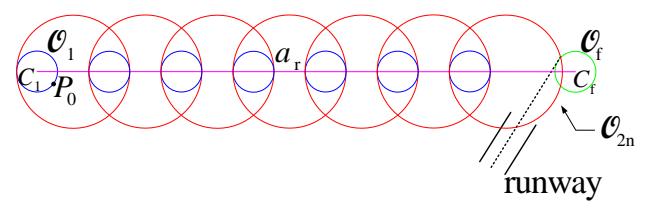

(b) $r_{1}<r_{2}$

Figure 6. Determination of $n_{m}$

Assume two radii of circular curves satisfy conditions 32 and 33 from Lemma IV.2 for all $r_{r}$. Since $J$ is dependent on $l_{r}, J=\left|2 r_{1}-2 r_{2}\right|$ for $a_{r}$ representing straight line. Then the Eq. 31 constraint becomes :

$$
\frac{\left\|\vec{r}_{c_{f} c_{i}}\right\|}{\left|2 r_{1}-2 r_{2}\right|} \leq n
$$

where $r_{1}$ and $r_{2}$ satisfy conditions 32 and 33 from Lemma IV.2. Since the lower bound in Eq. 37 is in $\mathbb{R}$, but $n$ is in $\mathbb{N}, n_{m}=\left\lceil\frac{\left\|\vec{r}_{c_{f} c_{i}}\right\|}{\left|2 r_{1}-2 r_{2}\right|}\right\rceil$ where $r_{1}$ and $r_{2}$ satisfy the conditions 32 and 33 from Lemma IV.2. By Theorem III.1, there exists $a_{r_{m}}$ such that $\mathcal{O}_{2 n}$ and $\mathcal{O}_{f}$ are tangent at $p_{2 n}$.

The $r_{r}$ of $a_{r} \in \mathcal{A}_{r}$ has range $\frac{\left\|\vec{r}_{c_{f} c_{i}}\right\|}{2 r_{r}}$ to $\infty$ according to $\lambda \in[-\infty, \infty]$. However, we have the following constraints on $r_{r}$ of $a_{r} \in \mathcal{A}_{r}$ from Eq. 24 along with $r_{r} \geq \frac{\left\|\vec{r}_{c_{f} c_{i}}\right\|}{2}$ :

$$
r_{r} \neq 0, \quad r_{r}>\frac{r_{1}}{2}, \quad r_{r} \geq \frac{\left\|\vec{r}_{c_{f} c_{i}}\right\|}{2}
$$

Note that $r_{1}$ may be greater than $\left\|\vec{r}_{c_{f} c_{i}}\right\|$ for some cases where $\lambda \in[-\infty, 0)$.

\section{Example Landing Trajectories Possible with the TDV Solution}

As with our previous work, we adopt a kinematic model to represent flight path displacements of the TDV, i.e. $\mathcal{B} .^{8}$ In these examples, we ignore the transition between two trim states under the assumption that transient disturbances can be compensated by the guidance and control system in a manner that restores the vehicle to the reference path relatively quickly after the transition is complete. Figure 7 illustrates the families of solutions with lateral plane paths designated by $\mathcal{B}=\left\{b_{i} \mid i \in\left\{1,2, \cdots, n_{m}\right\}\right\}$ and $a_{r_{m}}$ indicated by red dots. Figure 7 satisfies condition 32, and Figure 8 satisfies condition 33. Since the distance between two centers of the initial and final circular curves increases from (a) to (d), as shown in Figures 7 and $8, n_{m}$ also increases from (a) to (d). In Figure 7 (a) and (c), $\lambda$ of the reference arc having $n_{m}$ is in $[-\infty, 0)$. Note that $r_{1}>\left\|\vec{r}_{c_{f} c_{i}}\right\|$ in Figure 7 (a). Moreover, $\lambda$ of the reference arc having $n_{m}$ is in $[0, \infty]$, as shown in Figure 7 (b) and (d). Unlike the case where $r_{1}>r_{2}, \lambda \in[0, \infty]$ in Figure 7 (a) to (d). The selected runway can be reached by $\mathcal{B}$ with $a_{r_{m}}$, as shown in Figures 7 and 8 .

\section{Conclusions and Future Work}

This paper has introduced an analytic trajectory planning method in which minimum and maximumradius turning flight segments are sequenced to safely land a disabled aircraft that can no longer fly along a straight path. The proposed solution covers the comprehensive set of possible initial and final states in the lateral plane. This work complements a traditional Dubins path solver, providing a computationally-efficient (thus real-time) alternative composed only of turning flight segments. Our geometric solution is purposely simple, requiring only two trim states representing maximum and minimum turning radius maneuvers that 


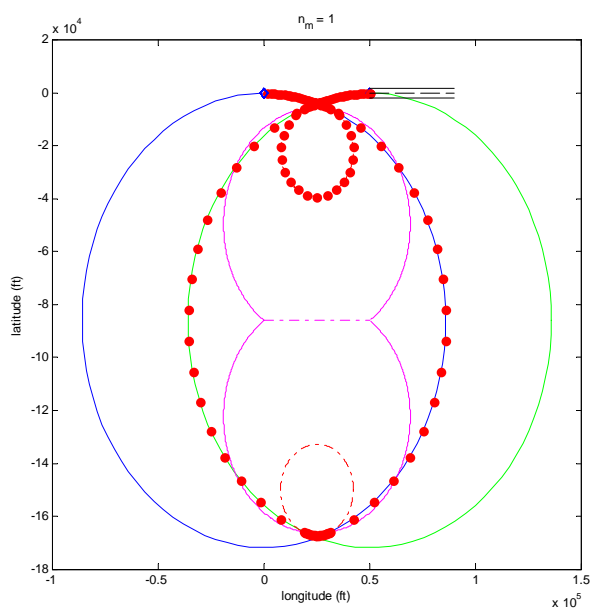

(a) $n_{m}=1$

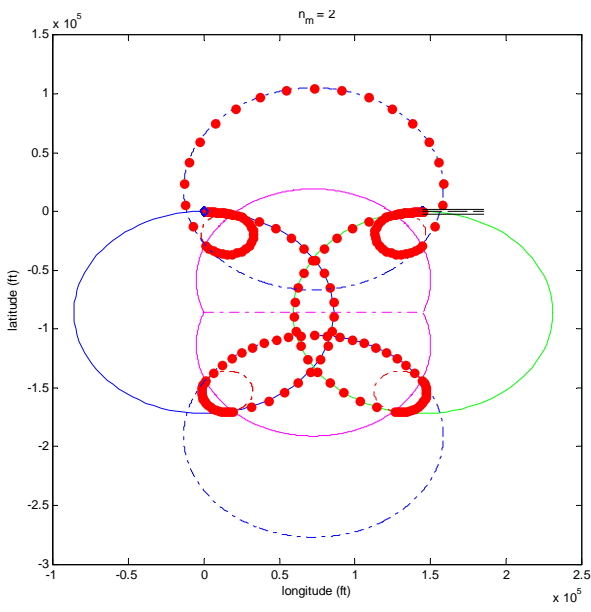

(c) $n_{m}=2$

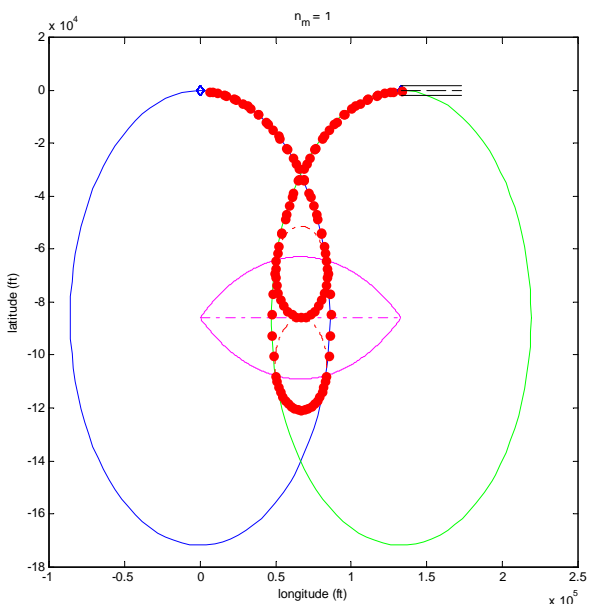

(b) $n_{m}=1$

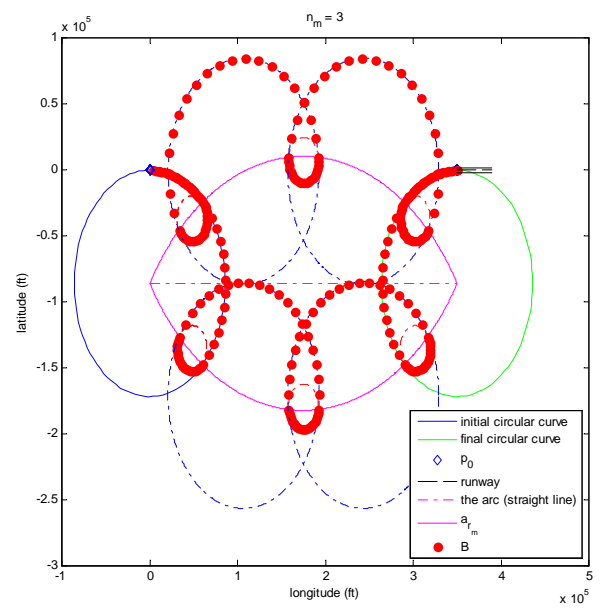

(d) $n_{m}=3$

Figure 7. Simulation Results for $\mathcal{B}$ when $r_{1}>r_{2}$

must include a safety factor appropriate for aircraft and environmental conditions. A reference arc $a_{r}$ defines the path along which a sequence of alternating minimum/maximum radius trimmed (steady) turns are followed.

The proposed analytic trajectory planner is highly computationally efficient compared with existing search-based methods, including that applied to the left wing damage case studied in our previous work. ${ }^{3}$ Without search, this work guarantees the shortest-length path to guide the aircraft to the desired touchdown state given the inability to fly straight. However, we currently make two simplifying assumptions that must be fully addressed in future work. First, we ignore configuration changes accumulated during the transition between trim states, i.e., from a state $i-1$ to $i$. Such transitions have been shown to be nontrivial but will require substantial effort to accurately represent in the purely geometric model we have developed in this work. Second, we presume the longitudinal and lateral aircraft dynamics are fully-decoupled, and that we can achieve a flight path angle that yields the necessary altitude change from initial to final (landing) state. Although this assumption has allowed analysis of landing trajectories strictly in the lateral plane, in future work constraints on flight path angle as a function of turning radius must be respected, potentially requiring an algorithm to automatically extend the minimum-length landing path generated by the TDV solver.

Ultimately, the task of the feedback control system will be to minimize the error between the planned and actual reference trajectory in inertial coordinates. This will require deviation from the "idealized" trim states used by our flight planner, with alterations particularly in reference bank, pitch, and thrust guiding the aircraft back to its inertial path as needed. With real-world disturbances such as wind or induced disturbances such as the ignored transitions between trim states, the centers of the alternating-radius turning sequences 


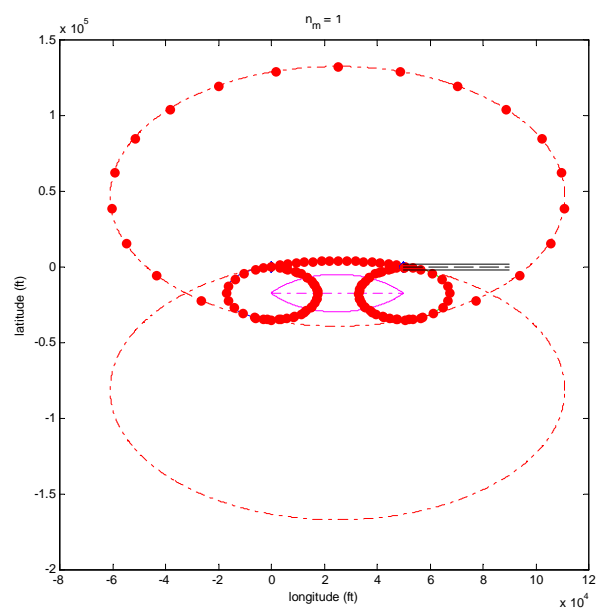

(a) $n_{m}=1$

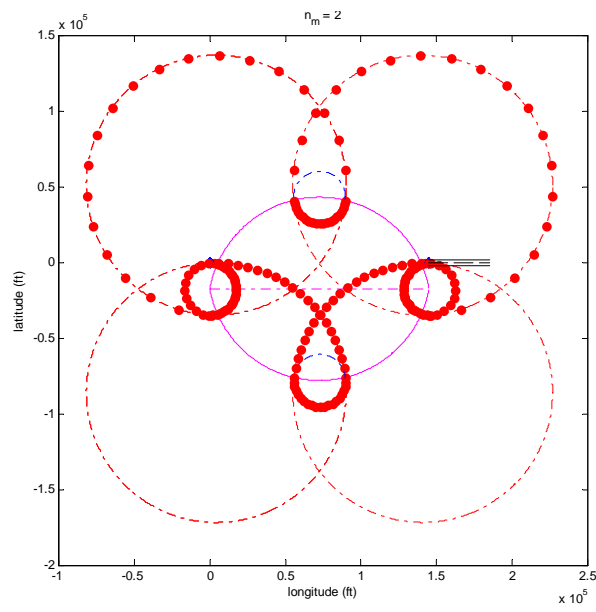

(c) $n_{m}=2$

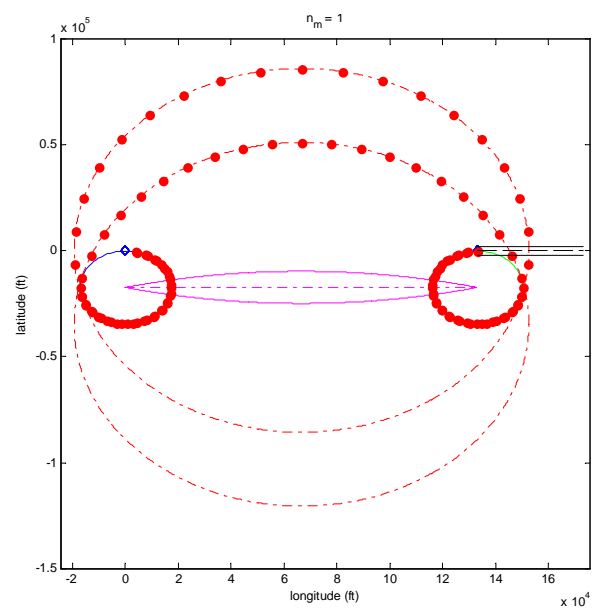

(b) $n_{m}=1$

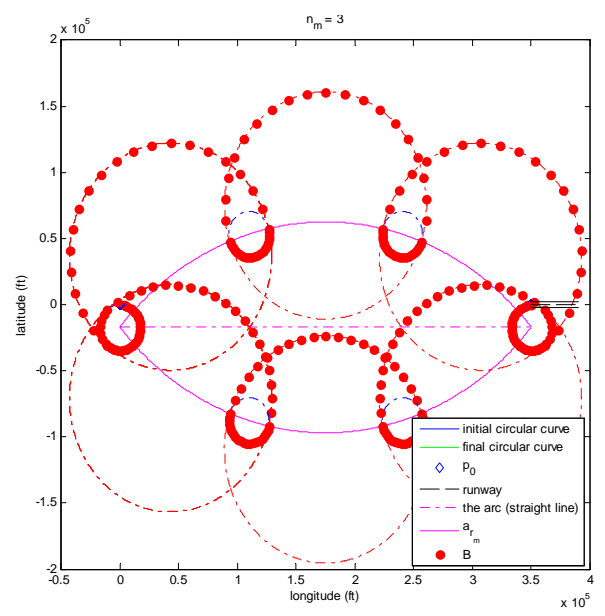

(d) $n_{m}=3$

Figure 8. Simulation Results for $\mathcal{B}$ when $r_{1}<r_{2}$

will be shifted in inertial space. Future work is required to anticipate, e.g., through fast-time simulation, situations under which the controller cannot fully-compensate for the suite of disturbances and adjust flight plans accordingly.

\section{References}

${ }^{1}$ Atkins, E., Portillo, A., and Strube, M., "Emergency Flight Planning applied to Total Loss of Thrust," Journal of Aircraft, Vol. 43, No. 4, Jul-Aug 2006, pp. 1205-1216.

${ }^{2}$ Strube, M., Sanner, R., and Atkins, E., "Dynamic Flight Guidance Recalibration after Actuator Failure," 1st AIAA Intelligent Systems Conference, Sep. 2004.

${ }^{3}$ Tang, Y., Atkins, E., and Sanner, R., "Emergency Flight Planning for a Generalized Transport Aircraft with Left Wing Damage," Proc. Guidance, Navigation, and Control Conference, Aug. 2007.

${ }^{4}$ Chen, T. and Pritchett, A., "Development and Evaluation of a Cockpit Decision-Aid for Emergency Trajectory Generation," Journal of Aircraft, Vol. 38, No. 5, Sep.-Oct. 2001, pp. 935-943.

${ }^{5}$ Saunders, J., Beard, R., and McLain, T., "Obstacle Avoidance Using Circular paths," AIAA, Guidance, Navigation, and Control Conference, Aug. 2007.

${ }^{6}$ Brinkman, K. and Visser, H., "Optimal Turn-Back Maneuver after Engine Failure in a Single-Engine Aircraft during Climb-Out," Journal of Aerospace Engineering, Vol. 221, No. 1, 2007, pp. 17-27.

${ }^{7}$ Boskovic, J., Prasanth, R., and Mehra, R., "A Multi-Layer Autonomous Intelligent Control Architecture for Unmanned Aerial Vehicles," J. Aerospace Computing, Information, and Communication, Vol. 1, 2004, pp. 605-628.

${ }^{8}$ Strube, M. J., Post-failure Trajectory Planning From Feasible Trim State Sequences, Master's thesis, Aerospace Engineering Dept., University of Maryland, College Park,, 2005. 